\title{
IMPACTO DE LA ESTRATEGIA METODOLOGICA DEL PROGRAMA ONDAS EN LA FORMACIÓN DE MAESTRAS Y MAESTROS
}

\author{
DIANA KATHERYNE ANDRADE MORENO *
}

\begin{abstract}
El trabajo que se presenta a continuación, pretende dar cuenta del impacto de la estrategia de formación de maestras y maestros pertenecientes al Programa Ondas en el Departamento del Huila, en cuatro aspectos fundamentales: académico, investigativo, metodológico y evaluativo.
\end{abstract}

\section{CONTEXTO}

El Programa Ondas, es la estrategia principal del Departamento Administrativo de Ciencia, Tecnología e Innovación, Colciencias, para fomentar la construcción de una cultura ciudadana y democrática de la ciencia, la tecnología y la innovación en la población infantil y juvenil colombiana, mediante la realización de investigaciones sugeridas por ellos y sus maestros donde los niños, niñas y jóvenes a través de la investigación, resuelven preguntas y cuestionamientos sobre su comunidad, su entorno geográfico y/o sus instituciones educativas "APRENDIENDO A INVESTIGAR INVESTIGANDO".

El Programa crea la Estrategia de Formación para Maestras y Maestros Ondas en busca de cualificar a estos profesionales y brindarles la oportunidad de ser parte de la calidad educativa desde la transformación de la práctica pedagógica a través de su metodología; la Investigación como Estrategia Pedagógica - IEP - la cual se define como "una alternativa para la búsqueda e implementación de nuevos procesos pedagógicos que respondan a los cambiantes tiempos educativos y favorezcan la elaboración de miradas críticas del mundo"( ); es decir, una propuesta que ve en la escuela - un escenario para formar en investigación a las culturas infantiles y juveniles desde temprana edad y convertir el conocimiento en útil y real - .

\section{SOBRE EL PROBLEMA DE INVESTIGACIÓN}

Los cambios de un mundo globalizado están íntimamente relacionados con el desarrollo de las comunidades y los momentos históricos que se han propiciado para favorecer la formación de los niños, niñas y jóvenes del mundo, donde el maestro toma otra posición.

Uno de los cambios más significativos del docente en el proceso educativo, ha sido dejar de lado su rol protagónico, jerárquico y restringido para convertirse en un guía, una pieza de la estructura que hace posible una educación de calidad.

El maestro investigador surge en la década de los 70 en Inglaterra, suponiendo una nueva concepción del maestro. Se le adjudica un papel activo en la renovación de la enseñanza para superar el papel de puro consumidor o aplicador de conocimientos. Se le concibe como un profesional crítico de su propia práctica y, en cierta medida autónomo, ejerciendo su profesión como investigador en el aula.

En este movimiento, la docencia, principal función del maestro, difícilmente se podrá llevar a cabo si no va a acompañada de un conocimiento reflexivo y crítico de su prác- 
tica profesional que se apoye en la investigación, proceso que debe desarrollarse en su realidad educativa: el aula.

La investigación entonces, se convierte en un elemento central y definitivo, es la estrategia que propicia el cambio en el paradigma formativo: donde surge una transposición de un proceso anclado en la enseñanza a un proceso cuya esencia es el aprendizaje. Por estas razones es creado el Programa Ondas, es el primer eslabón en la escala de formación del talento humano, su propósito es impulsar el conocimiento y la investigación a propagarse de manera flexible, para la creación de Redes, donde niños, niñas, jóvenes, maestras y maestros puedan tener actitudes científicas y tecnológicas que posibiliten la generación, validación y apropiación del conocimiento.

\section{ANTECEDENTES}

El Programa Ondas, fue acogido por Departamento del Huila desde febrero de 2006, mediante un convenio suscrito entre Colciencias, la Gobernación del Huila y la Universidad Surcolombiana, entidad que se ha encargado de la coordinación del Programa.

En consecuencia, de manera lenta pero progresiva, las Instituciones Educativas en el Departamento han adoptado esta estrategia y han promovido que sus docentes se ubiquen como puentes de conocimiento poco explorado, donde el maestro tiene al igual que los niños, la oportunidad de profundizar en la comprensión del desarrollo científico.

Es por esta razón, que la Asamblea Departamental del Huila mediante ordenanza $\mathrm{N}^{\circ}$ 017 de 2007 formaliza el Programa ONDAS como una política pública departamental que estipula la incorporación de manera sistemática y permanente del Programa a los planes y programas del Sector Educativo y a los PEI de cada Institución Educativa del Departamento.

Los convenios entre el departamento y el programa Ondas, se establecieron para hacer parte de las acciones sistemáticas y permanentes de coordinación y cofinanciación interadministrativa, incluidas en el Plan de Desarrollo Departamental y en el Plan Decenal de Educación.

Desde su germinación al día de hoy, el Programa ha logrado año tras año ampliar su campo de acción y apoyar más grupos de investigación, razón por la cual, se ha hecho significativo el número de maestras y maestros que han apropiado de la Investigación como Estrategia Pedagógica.

Dicha formación ha contribuido a que algunas experiencias investigativas se hayan convertido en experiencias pedagógicas significativas y que la envergadura de los procesos y las problemáticas abordadas hayan sido tenidos en cuenta para la participación en eventos locales, regionales, nacionales e internacionales.

Por todo lo anterior, se hace necesario establecer que las preguntas que guían esta investigación están relacionadas con determinar:

¿Cuál es el impacto académico, investigativo, metodológico y evaluativo de la estrategia de formación de maestros Ondas, en los maestros y maestras del departamento del Huila que han participado en el Programa? y ¿Cómo contribuye esta experiencia en su desarrollo personal y profesional? 


\section{METODOLOGÍA}

La investigación fue de corte cualitativo, ya que permitió identificar visión y apropiación de la estrategia de formación y se enmarcó en la investigación evaluativa, que para esta propuesta se traduce en la evaluación de la conceptualización, el diseño, la implantación y la utilidad de la estrategia de formación a maestros y maestras en el departamento del Huila, a través de las siguientes categorías de análisis: Práctica Pedagógica, Investigación como Estrategia Pedagógica y Percepción de la Práctica Docente de los maestros Ondas.

La investigación contó con una audiencia específica de 25 maestros, equivalente a un porcentaje representativo de los maestros y maestras Ondas con trayectoria en el departamento del Huila, cuyo único criterio de selección hizo referencia a más de tres años de participación continua en el desarrollo de investigaciones abiertas y/o preestructuradas en el Programa.

\section{CARACTERIZACIÓN DE LOS MAESTR@S}

Los hombres y mujeres que hicieron parte de este estudio son profesionales pertenecientes a diferentes áreas de la pedagogía; entre las que se pueden precisar las licenciaturas en ciencias naturales, biología y física, ciencias sociales, matemáticas, lengua castellana y educación física. De igual manera, aunque en menor proporción, participan profesionales de áreas no ligadas a la pedagogía como la ingeniería agrícola y la comunicación social.

Estos profesionales cuentan además, con diversos títulos de especialización y algunos con Maestrías; las cuales hacen parte de los campos de las ciencias sociales, la ecología y la investigación en el currículo.

Estos maestros y maestras Ondas, laboran en su gran mayoría en las Instituciones Educativas de carácter público, desempeñándose tanto en la zona rural como urbana. Además, la gran mayoría están cobijados bajo el nuevo estatuto de profesionalización docente a través del decreto 1278(2).

Los maestros Ondas, además de incentivar el gusto por la investigación en sus estudiantes, favorecen el desarrollo de normas y valores, identificando al Grupo como una nueva familia que lo amerita.

Los maestros de este estudio se encuentran ubicados en 12 de los 37 municipios del Departamento. La experiencia en la docencia, se establece por la diferencia de edad de los participantes que es diversa.

Por otra parte, la gran mayoría señala que su experiencia en investigación, está ligada a las exigencias académicas de la Educación Superior a través de los Programas de formación en los que han participado.

De los 25 maestros que hicieron parte de este estudio 10 son mujeres y 15 son hombres. Lo cual nos da como primera conclusión que el género que más predomina por antigüedad o trayectoria en el Programa es el masculino.

\section{DESDE LAS CATEGORÍAS DE ANÁLISIS: ¿QUÉ ES EL PROGRAMA ONDAS PARA LOS MAESTROS ONDAS HUILA?}

Los maestros y maestras Ondas Huila, definen el Programa como una oportunidad, 2 Decreto 1278 de junio de 2002. Ministerio de Educación Nacional. Estatuto de Profesionalización docente. Disponible en www.mineducacion.gov.co. 
precisando, que es la base de un camino para el relevo generacional de los científicos del país, que se construye poco a poco pero a diario.

Lo anterior se puede evidenciar en cada campo de la siguiente manera:

En lo académico: Porque transforma la realidad educativa, ya que promueve la formación de maestros y estudiantes simultáneamente a través de una estructura lógica, coherente y sencilla, que hace divertido el proceso de investigación.

En lo metodológico: Permite sustentar los procesos de enseñanza y aprendizaje desde la articulación teoría y práctica.

En lo investigativo: Porque se responde a las características del contexto en el cual se realiza su ejercicio docente.

En lo evaluativo: Por el Impacto de su acción educativa, ya que permite la evaluación periódica de el proceso y conlleva a la apropiación y divulgación de la indagación en la Institución, la comunidad y otros escenarios académicos.

\section{¿POR QUÉ PARTICIPAR?}

Se puede evidenciar que los maestros y maestras Ondas Huila, que hicieron parte de este estudio, se vincularon por:

En lo académico: Por su amor por los temas de ciencia, tecnología e innovación y su compromiso con la educación de las culturas infantiles y juveniles a su cargo.

En lo metodológico: Por desarrollar espacios para el diálogo de saberes y la negociación cultural.

En lo investigativo: Por estar a la vanguardia de nuevas estrategias $y / 0$ programas que redunden en prácticas pedagógicas que se centren en el niñ@, joven y/o estudiante.

En lo evaluativo: Porque en la medida que se forman crecen personal y profesionalmente.

\section{¿CÓMO SE PERCIBEN LOS MAESTROS ONDAS?}

Los maestros y maestras que participan en el Programa Ondas y que apropian la Investigación como Estrategia Pedagógica; tienen como palabra clave para auto describirse como: "distintos o diferentes" pero ante todo: Comprometidos con la educación.

En primera instancia, el formarse para vivir la docencia de forma lúdica, práctica y real promueve identidad y le devuelve al docente y a la profesión, el estatus que ha perdido con el paso de los años. Por esta razón, cuando los maestros Ondas perciben aceptación, reconocimiento y que sus estudiantes se han transformado, sienten que han aportado el granito de arena para contribuir a los cambios que exige una educación globalizada.

En lo académico: Por volver la investigación parte de su discurso y conducir toda su práctica pedagógica al diálogo con sus estudiantes a partir de interrogantes, a la vivencia, al descubrir y al proponer estrategias.

3 Amor, entendido como fascinación, gusto, curiosidad. 
En lo metodológico: Por ser profesionales capaces de afrontar las exigencias administrativas, (dirección de cursos, diligenciamiento de formatos, entre otros), y a la vez procurar espacios de formación integral desde la investigación.

En lo investigativo: Por promover la capacidad de asombro de sus estudiantes, por acercar y desmitificar el mundo científico.

En lo evaluativo: Por aprender haciendo, cambiar su rol y poder reflejar esto en su vida personal.

\section{¿QUÉ CAMBIOS GENERA?}

Para los maestros y maestras que realizan investigación a partir de las preguntas que se formulan los niños, niñas y jóvenes; los cambios, están a la orden del día y es todo un cambio en la imagen y concepción del docente que ellos tenían y que tiene arraigada la comunidad educativa.

En lo académico: Por contribuir a la formación de jóvenes capaces de leer el contexto, analizarlo e interpretarlo para plantear alternativas de solución a los múltiples problemas que los rodean, contribuyendo de esta manera a tener personas críticas y con sentido de pertenencia e identidad.

En lo metodológico: La IEP permite transversalizar el proceso de enseñanza - aprendizaje y promover que el conocimiento esté articulado con cada área.

En lo investigativo: Por contribuir a la formación del espíritu científico.

En lo evaluativo: Un crecimiento total, porque enfocan su vida personal y profesional desde lo investigativo.

\section{¿CUÁLES SON LOS APORTES DE ESTA EXPERIENCIA?}

Para los maestros y maestras Ondas, los aportes son más de tipo personal, puesto que hacen parte de un cumulo de experiencias que los ayudan a crecer, mejorar e ir más allá, más allá de lo instruccional, jerárquico y tradicional.

Entre los aportes más significativos vale la pena destacar los siguientes:

1. Se convierten en referentes y modelos a seguir de sus compañeros, quienes los alaban por la dedicación y responsabilidad con la que sumen el Programa, el cual les exige tiempo extracurricular para la planeación y la indagación con su Grupo, diálogo permanente con las directivas y los padres familia y presentación y/o divulgación de resultados.

2. Obtienen meritos y reconocimientos en otras Entidades que además apoyan su labor y trabajo con el Grupo.

3. Son referentes en la evaluación institucional cuando se precisa el componente investigativo.

4. Son los maestros más queridos y admirados de las IE a las que pertenecen por los estudiantes.

5. Son los maestros elegidos para ser parte de Programas y Eventos a nivel local, regional y nacional. 


\section{¿CUÁLES SON LAS DIFICULTADES?}

Aunque los maestros y maestras Ondas Huila se consideren y sean distintos, no son ajenos a las fallas de un sistema que avanza pero lentamente y precisan como dificultades las siguientes:

1. No hay reconocimiento por parte de los entes gubernamentales y de las directivas institucionales.

2. No hay espacios para desarrollar investigación dentro de la asignación académica.

3. No siempre cuentan con apoyo de la comunidad educativa para realizar las indagaciones.

\section{¿QUÉ HACE FALTA?}

Para los maestros que hicieron parte de este estudio manifiestan que es necesario:

1. Apoyo de la Secretaría de Educación y por ende de sus entes gubernamentales para que Programas como Ondas tengan mayor acogida.

2. Integrar los procesos investigativos desarrollados por el programa Ondas con los PEI de las Instituciones Educativas como política institucional, para facilitar el desarrollo de las investigaciones llevadas a cabo por los grupos de investigación.

3. Contar con colaboración interinstitucional de universidades y otras entidades, para garantizar apoyo y emprendimiento, a proyectos que pueden convertirse en valiosas e innovadoras empresas.

4. Aumentar el apoyo económico que se les brinda a los Grupos de Investigación para la realización de sus indagaciones, debido a que el dinero no les alcanza, sobre todo a quienes se encuentran en zonas apartadas del departamento.

5. Que cambien la política de cobertura para el número de integrantes por Grupo de investigación para no masificarla.

\section{CONCLUSIONES}

De acuerdo al análisis realizado, a partir de la información obtenida de las fuentes y con la aplicación de los instrumentos; se puede precisar que los maestros con trayectoria son los profesionales ejemplo a seguir de la educación en el departamento. Es admirable la entrega que tienen para promover éste tipo de estrategias, que lamentablemente no ofrecen más beneficios que la satisfacción personal y el reconocimiento de su comunidad educativa.

La metodología de la Investigación como Estrategia Pedagógica IEP es válida, tiene contemplado cada aspecto y cuando es llevada a cabo bajo el rigor con el que la han asumido estos maestros, se pueden apreciar las transformaciones de estos profesionales de la educación, tanto en lo personal como el campo laboral.

La apropiación de la IEP en estos maestros, es de resaltar, debido a que conocen los espacios, manejan el material y para referirse a la aplicación de la metodología, lo hacen utilizando el lenguaje científico de ésta estrategia.

Si se mide el impacto por la cantidad de maestros con trayectoria, el Programa ha fallado, pero teniendo en cuenta de que se trata de una estrategia en crecimiento, el Programa ha logrado permear en una población importante que puede evidenciar grandes resultados. 
Pese a que la educación es un pilar y es la razón de ser de un país, las condiciones en las que se desempeñan nuestros maestros distan de las buenas intenciones y es de resaltar que el Programa se convierte en una oportunidad para salir de la monotonía y soñar con tener una oportunidad más allá de su vereda y /o municipio.

Aún hace falta arraigar el Programa en el departamento y promover que todos y cada uno de los miembros de la comunidad educativa del Huila lo acoja y apoye.

El Programa debe brindar más beneficios a los maestros, con el ánimo de garantizar su continuidad y motivar a más maestros a participar en él.

Es triste que un Programa de la envergadura de Ondas, la política lo convierta en cifras para la masificación y la cuantificación de resultados y deje de ser la apuesta por fascinar a las culturas infantiles y juveniles en la investigación, lo cual contrasta en las oportunidades que el mismo brinda, es decir, para la participación de sus integrantes en los eventos.

\section{ALTERNATIVAS DE INTERVENCION}

De acuerdo a los resultados obtenidos de la presente investigación, se precisan los siguientes elementos como estrategias que se conviertan en aportes en los campos: académico, investigativo, metodológico y evaluativo de la estrategia del Programa.

1. Consolidar el proceso de formación de los maestr@s Ondas, procurándoles temas y espacios que contribuyan a su crecimiento personal y profesional y que por ende, apoye sus procesos de actualización y/o formación continuada como especializaciones y maestrías. En primer lugar se pretende que el Programa ofrezca conferencias de autoridades en el tema de investigación para los maestros Ondas, con lo cual, se propone ir más allá de la Guía de Investigación "Xua, Teo y sus Amigos en la Onda de la Investigación y la Caja de Herramientas para maestros Ondas" para presentarles temas, teorías e investigaciones entre otras, que permita poner en contexto y concatenar la Investigación como Estrategia Pedagógica con la práctica investigativa que se realiza en la Universidad.

En segundo lugar, y para consolidar el proceso de autoformación, se hace necesario procurarles un material pedagógico que se construya con los aportes de los Investigadores de la Universidad Surcolombiana, que les permita cimentar conceptos, métodos y teorías propias de la práctica investigativa. En tercer lugar que las publicaciones que se realicen de las investigaciones Ondas tengan el código que les permite registrarlo en su hoja de vida. Lo cual les asegura reconocimiento.

2. El proceso de formación y acompañamiento debe seguir promoviendo la utilización de las TICS, para que los maestros propendan por el uso, manejo y aprovechamiento de las Tecnologías de la Información y la Comunicación en su práctica pedagógica.

De esta manera, se garantizará que los maestr@s incursionen en las redes sociales y empiecen a manejar el lenguaje digital de los chicos, con lo cual se favorezcan las relaciones horizontales y se promueva el trabajo investigativo desde la red y que se convierta en otro espacio para el diálogo de saberes y la negociación cultural. Para ello es necesario alfabetizar a un buen número de docentes en el uso de las TICS y crear las plataformas para interacción entre maestros co investigadores.

3. Crear más espacios de interacción entre maestros y grupos investigadores para que la apropiación social del conocimiento consolide competencias investigativas. Con este proceso se busca fortalecer la cadena formativa del talento humano desde los forma- 
dores de las nuevas generaciones, es decir, que la retroalimentación que realicen los maestros entre ellos y con Investigadores pertenecientes a los Grupos de Investigación de la Universidad Surcolombiana generen competencias y habilidades que les sean transmitidas a los niños, niñas y jóvenes investigadores Ondas.

4. Crear espacios para vincular a la comunidad educativa y privilegiar el proceso de apropiación de la IEP. El primer paso para lograr este vínculo es a partir de la convocatoria, la cual no debe ser exclusiva del Grupo (maestro y estudiantes) sino que debe ser de la Institución Educativa y sus estancias, para que a través de los mecanismos formales de participación, se invite a la comunidad educativa y se les presente el Programa, sus objetivos, forma de trabajo y de participación.

5. Apoyar la realización de investigaciones de los maestros acerca de su experiencia como acompañante co investigador. Es necesario hacer una transición del docente co-investigador al docente investigador, por ésta razón y aprovechando el vínculo que se propicie con los Grupos de Investigación de la Universidad Surcolombiana; se propone que éstos apoyen con asesoría especializada la realización de pequeñas investigaciones de los maestros Ondas, con el propósito que favorezcan su práctica pedagógica y a la vez, brinden a los Grupos de Investigación aportes a sus líneas de investigación, que se conviertan a la postre en insumos de sus cátedras para los futuros profesionales de la educación.

6. Apoyar la vinculación de los maestros Ondas a los Grupos de Investigación, para darle continuidad a su formación. Se propone establecer con los Grupos de Investigación de la Universidad Surcolombiana unos criterios para permitir la vinculación de los maestros Ondas a sus Grupos, donde puedan apoyar sus desarrollos.

\section{REFERENTE DOCUMENTAL}

Aguirre Baztán, A. (coord.) Etnografía. Metodología cualitativa en la investigación sociocultural. Barcelona, Marcombo. 1995

Colciencias, Niños, niñas y jóvenes investigan. Lineamientos pedagógicos del Programa Ondas, Bogotá, COLCIENCIAS - Programa Ondas, Fundación FES, ICBF, 2006, p. 13.

Colciencias. Caja de Herramientas, Cuaderno No. 2, Lineamientos de la Estrategia de formación de maestros (as) Ondas, Bogotá, 2007, pág. 46

Colciencias, Universidad Externado de Colombia. Evaluación del impacto del programa Ondas. Bogotá. Citado por: Programa Ondas de Colciencias. Niños, niñas y jóvenes investigan. Lineamientos pedagógicos del Programa Ondas. 2005.

Dimaté Rodríguez, Cecilia. La ciencia, la tecnología y la innovación en las culturas infantiles y juveniles de Colombia. Evaluación de Impacto del Programa Ondas.

López Jiménez, Nelson Ernesto. La de- construcción curricular. Cooperativa Editorial Magisterio, 2001.

Mejía Jiménez, Marcos Raúl. La pregunta: entre estrategia pedagógica y camino investigativo. Construyendo rutas de saber y conocimiento. Revista Internacional Magisterio. 2007.

Mejía, Marco Raúl. Educación(es) en la(s) globalización(es) I. Entre el pensamiento único y la nueva crítica. Bogotá. Ediciones Desde Abajo. 2006. 